\title{
Investigating Flame Fronts in Competitive Exothermic Reactions
}

\author{
Z. Huang ${ }^{\text {a }}, \underline{\text { H. S. Sidhu }}{ }^{\text {a }}$ I. N. Towers ${ }^{a}$, Z. Jovanoski ${ }^{\text {a }}$ and V. V. Gubernov ${ }^{b}$ \\ ${ }^{a}$ Applied and Industrial Mathematics (AIM) Research Group, School of Physical, Environmental and \\ Mathematical Sciences, University of New South Wales at the Australian Defence Force Academy, Canberra, \\ Australia \\ bI.E. Tamm Theory Department, P.N. Lebedev Physical Institute, 53 Leninsky Prospect, 119991 Moscow, \\ Russia \\ Email: $\underline{\text { H.Sidhu@adfa.edu.au }}$
}

\begin{abstract}
The phenomenon of combustion is undoubtedly one of the most important physiochemical processes in nature and industry. Combustion theory has played a significant role in the advance of human civilization and provided an understanding of natural phenomena such as energy production from burning fossil fuels, the synthesis of advanced materials (e.g. the steady travelling wave is extremely important in the combustion synthesis via the Self-propagating High-temperature Synthesis process) and bushfire (the combustion models can be utilized to give correct predictions or even effective control over mountainous or grassland bushfires). However, there are still many aspects of combustion that are still not understood. Hence, there is a great value in understanding the process of combustion through mathematical modelling.

In this work, we consider travelling wave solutions of a one-dimensional reaction-diffusion system corresponding to two competitively exothermic reactions with the inclusion of Newtonian heat loss. We suppose all reactions occurring during the combustion may be lumped together as two different paths. The two exothermic reactions compete for the same reactant and both reactions are well described by the Arrhenius law. Such a reaction scheme has been shown to represent the combustion of titanium-carbon-hydrogen and zirconiumcarbon-hydrogen experimentally. The model presented considers a general case of there being volumetric heat loss transferring heat to the surroundings in the reactions.

Properties of travelling wave fronts, particularly wave speed, are determined numerically by solving the governing partial differential equations (PDEs). The speed of the combustion wave is analysed for different values of the heat loss parameter with other parameter values fixed. We point out that as the heat loss coefficient increases with other parameter values fixed, the flame speed decays gradually before the front ceases to exist. This is expected as increasing the heat loss would result in less energy being available to sustain the propagation of the flame fronts. We also determine how the extinction limit depends on the heat loss parameter as well as the exothermic parameter. Numerical solutions show that there is no travelling wave when we choose the parameter values of the exothermicity and heat loss parameter above threshold values. All numerical solutions were obtained using the Method of Lines approach to solve the governing PDEs and were independently verified using a commercial finite-element package, FlexPDE ${ }^{\mathrm{TM}}$.
\end{abstract}

Keywords: Combustion, two-step competitive exothermic reactions, heat loss, travelling wave, wave speed 


\section{INTRODUCTION}

Combustion technology has numerous practical applications in many aspects of our lives from power generation to the synthesis of advanced materials. An example of the latter is the self-propagating high-temperature synthesis (SHS) which has already been used in aerospace and hi-tech manufacturing (Merzhanov, 1990). One-step irreversible exothermic reaction models such as (1) have led to a great many useful and qualitatively correct predictions, and contributed significantly to the understanding of combustion phenomena. In such a scheme, it is assumed that fuel is directly converted into product and heat is released in a single reaction. A general schema of such reaction scheme can be represented as

$$
A \stackrel{k(T)}{\longrightarrow} B+Q
$$

where $A$ is the fuel, $B$ the product, $Q$ the heat released by the reaction and $k(T)$ represents the reaction rate.

However, combustion processes generally involve hundreds or even thousands of physical and chemical reactions. It is no longer reasonable to utilize a single composite reaction model to study all kinds of combustion processes. In fact, Westbrook and Dryer (1981) has stated that models with simplified one-step mechanisms can yield erroneous results. Detailed multi-step models have been developed to analyse the properties of propagating wave front solutions. Although recent advances in computation power is possible to investigate the flame behaviour utilizing equations of energy and mass transfer for all of species involved with detailed mechanism and such investigations have provided some quantitative results for observed phenomena, there is still plenty of uncertainty when applied to study the generic behaviour of flames. Fortunately, much understanding can be gained by considering reduced kinetic mechanisms that reproduce the essential phenomenology. For instance, Bechtold and Law (1994), Seshadri et al. (1994) and Sánchez et al. (1996) reduced the detailed schemes of the hydrogen and methane oxidation involving dozens of intermediate reactions to several steps. Sánchez et al. (1996) have pointed out that the 'lumped' models are able to produce excellent quantitative results.

In this paper we focus on the investigation of premixed combustion waves in a model with two-step competing exothermic reactions, where the same reactive material provides the feed for both reactive steps. As reported by Martirosyan et al. (1983a,b), such a reaction has direct relevance to the combustion of Me-C- $\mathrm{H}_{2}$, where Me is either Ti or Zr. Sidhu et al. (2013), Towers et al. (2013) and Towers et al. (2013) have investigated flame propagation in a model with two-stage competitive exothermic reactions (exo-exo reactions) and demonstrated the existence of regions of bi-stability as well as pulsations under adiabatic conditions. The work reported here extends the above work to take heat loss into consideration, i.e. non-adiabatic conditions.

\section{GOVERNING EQUATIONS}

We consider a model in one dimension that involves two independently irreversible exothermic reactions:

$$
A \stackrel{k_{1}(T)}{\longrightarrow} B+Q_{1}, \quad A \stackrel{k_{2}(T)}{\longrightarrow} C+Q_{2}
$$

where $A$ represents the reactant; $B$ and $C$ are chemically inert products and do not change their physical properties such as density, heat capacity nor the diffusivity of the system during the whole combustion process; $Q_{1}$ and $Q_{2}$ describe the heat released by the first reaction and by the second reaction respectively. Here we assume neither $Q_{1}$ nor $Q_{2}$ is less than zero since only the two-stage competitive exothermic reactions scheme is of interest in this investigation. The two reaction rates $k_{1}(T)$ and $k_{2}(T)$, assumed to be highly dependent on the reaction temperature $T$, obey Arrhenius kinetics and take on the form

$$
k_{i}(T)=A_{i} e^{-\frac{E_{i}}{R T}}
$$

where $A_{i}$ and $E_{i}$ are the first-order rates and the activation energies for the respective reaction $i=1,2$. The universal gas constant is represented by $R$. The nonadiabatic governing partial differential equations (PDEs) derived from the conservation of energy and fuel mass are

$$
\begin{aligned}
\rho c_{p} \frac{\partial T}{\partial t} & =k \frac{\partial^{2} T}{\partial x^{2}}+\rho Q_{1} Y k_{1}(T)+\rho Q_{2} Y k_{2}(T)-\frac{h S}{V}\left(T-T_{a}\right) \\
\frac{\partial Y}{\partial t} & =D \frac{\partial^{2} Y}{\partial x^{2}}-Y k_{1}(T)-Y k_{2}(T)
\end{aligned}
$$


Here $T$ is the temperature and $Y$ represents the fuel concentration, $\rho$ is the fuel density, $c_{p}$ is the specific heat, $k$ is the thermal conductivity, $D$ represents the fuel diffusivity, $h$ is the heat transfer coefficient from fuel to the ambient surroundings, $S / V$ represents surface area to volume ratio, $T_{a}$ is the ambient temperature. The Newtonian cooling is modelled using the term $\frac{h S}{V}\left(T-T_{a}\right)$ in the energy balance equation (3).

On introduction of the dimensionless variables

$$
\tau=\frac{Q_{2} A_{2} R}{c_{p} E} t, \quad \xi=\sqrt{\frac{\rho Q_{2} A_{2} R}{k E_{2}}} x, \quad u=\frac{R T}{E_{2}},
$$

the governing PDEs can be written as

$$
\begin{aligned}
\frac{\partial u}{\partial \tau} & =\frac{\partial^{2} u}{\partial \xi^{2}}+v e^{-1 / u}+q r v e^{-f / u}-l\left(u-u_{a}\right) \\
\frac{\partial v}{\partial \tau} & =\frac{1}{L e} \frac{\partial^{2} v}{\partial \xi^{2}}-\beta v e^{-1 / u}-r \beta v e^{-f / u}
\end{aligned}
$$

where

$$
L e=\frac{k}{\rho c_{p} D}, \quad f=\frac{E_{1}}{E_{2}}, \quad q=\frac{Q_{1}}{Q_{2}}, \quad r=\frac{A_{1}}{A_{2}}, \quad l=\frac{h S E_{2}}{V R \rho Q_{2} A_{2}} \quad \text { and } \quad \beta=\frac{c_{p} E_{2}}{Q_{2} R}
$$

The dimensionless temperature and mass fraction are denoted by $u$ and $v$ respectively; $\xi$ and $\tau$ represent non-dimensional space and time coordinates. The parameters $q, r$ and $f$ are the ratios of enthalpies, the pre-exponential factors and activation energies, respectively. The exothermicity parameter $\beta$ is the ratio of activation energy to heat released by the second reaction. The parameter $l$ represents the volumetric heat loss coefficient. The Lewis number, $L e$, is the ratio of thermal conductivity to mass diffusivity, varying from around unity for gaseous fuel to infinite for solid fuel as noted in Williams (1985) and Zeldovich et al. (1985). For example, the Lewis number $L e$ is around unity for gaseous fuels (methane has $L e \approx 1.002$ and $50 \% \mathrm{CO}+\mathrm{Air}$ has $L e \approx 1)$ and $L e \rightarrow \infty$ for solid fuels $\left(\mathrm{H}_{2}-\mathrm{Fe}\right.$ has $\left.L e \approx 3.346 e 8\right)$.

The governing equations are subject to the boundary conditions

$$
\begin{aligned}
& u=u_{a}, \quad v=1, \text { for } \xi \rightarrow \infty \\
& u=u_{a}, \quad \frac{\partial v}{\partial \xi}=0, \text { for } \xi \rightarrow-\infty
\end{aligned}
$$

On the right boundary $(\xi \rightarrow \infty)$, we have a 'cold' and 'unburnt' state (the dimensionless ambient temperature is denoted as $u_{a}$ and the consumption of the fuel is negligible). For definiteness and without loss of generality, the initial fuel concentration is chosen to be 1 . On the left boundary $(\xi \rightarrow-\infty)$, the temperature will cool down to the ambient temperature. However, the fuel concentration can not be specified. No reaction occurs at this steady state, and the derivative of $v$ is, therefore, set to zero.

\section{NUMERICAL SOLUTIONS}

The results presented in this paper were obtained via solving the governing partial differential equations (6-9) using a semi-discrete method - - the method of lines (Schiesser (1991)). Method of Lines (MOL) has been one of standard tools for solving partial differential equations (PDEs) since 1970s. MOL reduces a boundary value problem to a system of ordinary differential equations (ODEs) in time through discretization in space. Then a variable time-step approach with time local error control can be employed to solve the resulting ODE system. It is regarded as a special finite differential method and a semi-analytical method since it has the merits of both the finite difference method and analytical method.

From the viewpoint of efficiency and accuracy, we set the number of the mesh nodes to be 20000 along the $\xi$-axis, over the interval $0<\xi<8000$, and time span to $\tau \in[0,1000]$ to ensure that a stable propagating front has been established. The initial mass fraction was set to the maximum value $v(\xi, 0)=1$, whereas the temperature was initially taken to be a simple Gaussian profile, $u(\xi, 0)=A \exp \left(-\xi^{2}\right)$, where $A$ is a constant. The ODE solver 'ode15s', a stiff ODE solver provided in Matlab ${ }^{\circledR}$, is used to solve the system of ODEs. Since this is a preliminary investigation of this model with the inclusion of heat loss, we shall fix the values of the Lewis number for the fuel to $L e=2$, the ratios of the activation energies, the pre-exponential factors and 
the heats, $f=1.5, r=25, q=5$ following Towers et al. (2013). Without loss of generality and following the work of Weber et al. (1997), we fix the ambient temperature $u_{a}=0$ throughout this paper. The main aim of our work is to investigate the properties of the travelling wave solutions, in particular the propagating wave speed, and how it varies as the exothermicity parameter $\beta$ and the heat loss parameter $l$ vary. We also used FlexPDE ${ }^{\mathrm{TM}}$ to independently verify our solutions from MOL. FlexPDE ${ }^{\mathrm{TM}}$ is a commercial finite-element package for solving partial differential equations in one, two and three dimensions to obtain steady state or time-dependent solutions. It allows users to set up the relative error tolerance. Whenever this prescribed error tolerance is reached or exceeded, the mesh size is refined and solutions are recalculated until the estimated errors are within the set tolerance level. In this paper, we set the error tolerance limit to errlim $=0.0001$.

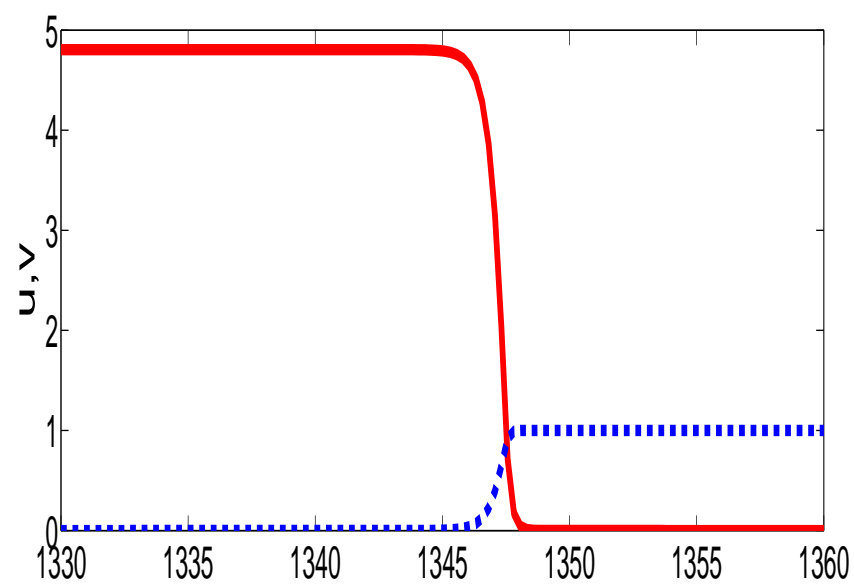

Figure 1. Combustion wave front solution profiles for temperature (red solid line) and fuel concentration (blue dashed line) for the adiabatic case with $\beta=1.0, L e=2, q=5, r=25$ and $f=1.5$. The horizontal axis represents the coordinate in the moving frame of the front, $\xi$.

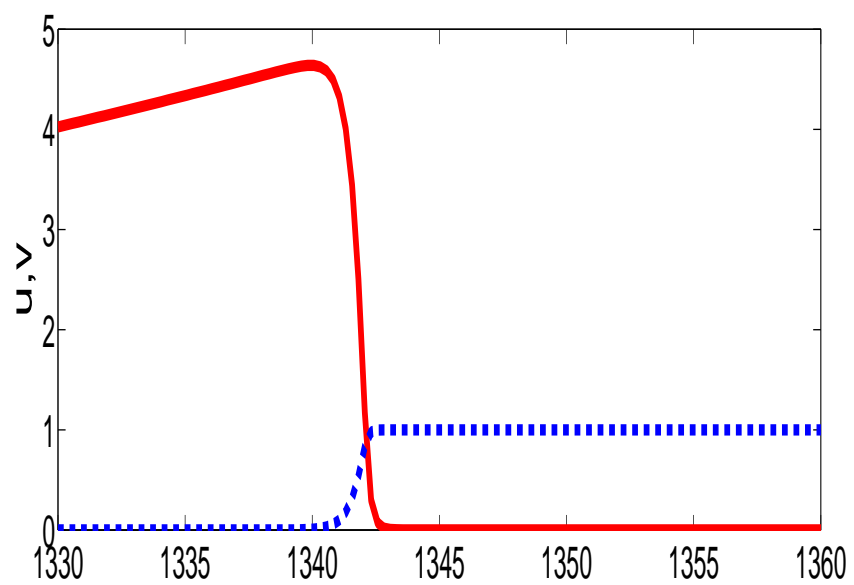

Figure 2. Combustion wave front solution profiles for temperature (red solid line) and fuel concentration (blue dashed line) for the non-adiabatic case. Here the heat loss coefficient $l$ is chosen to be 0.1 and other parameter values are the same as those given in Figure 1.

Figures 1 and 2 demonstrate the travelling wave profiles for the adiabatic and non-adiabatic cases respectively. The wave moves from the left ('hot' and 'burnt' region) to the right ('cold' and 'unburnt' region). The profiles 
of the adiabatic and non-adiabatic cases are similar. The main difference is that the temperature profile in Figure 2 has 'a cooling tail' after reaching the peak for the non-adiabatic case while in figure 1 it remains unchanged since no heat is lost to the surroundings. It should be also noted that the temperature in figure 2 must cool down to the ambient temperature behind the front. On the other hand, the profiles for fuel concentration in Figure 1 and 2 seem to be similar. However, we point out that there is some residual amount of fuel left behind the travelling wave front for the non-adiabatic case although the leftover is too small to be noticed in Figure 2. We note that all the fuel is consumed in the adiabatic case.

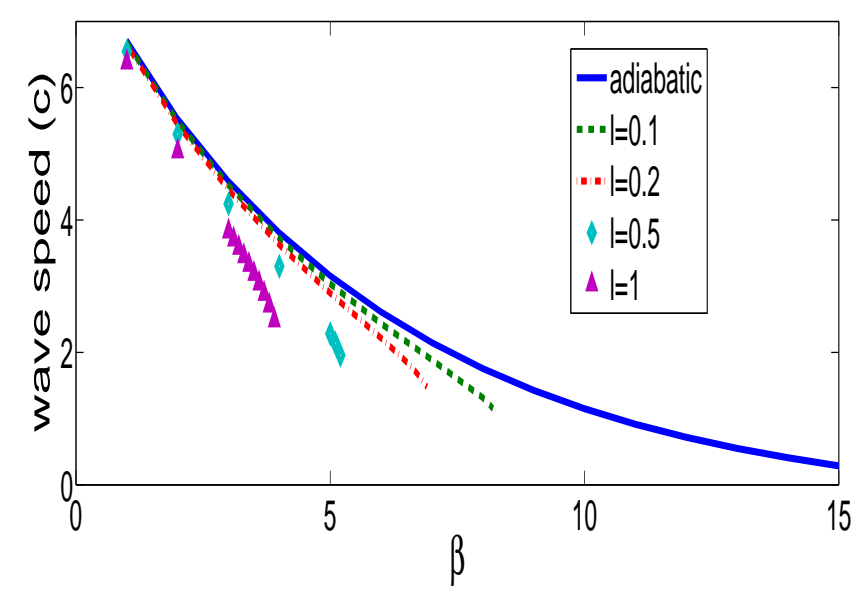

Figure 3. Wave speed versus $\beta$ for five different values of the heat loss coefficient: Solutions for $l=0, l=$ $0.1, l=0.2$ are separately represented by the blue solid line, green dashed line and red dash-dot line, whereas the cyan diamond and the magenta triangle correspond to solutions for $l=0.5$ and $l=1$, respectively.

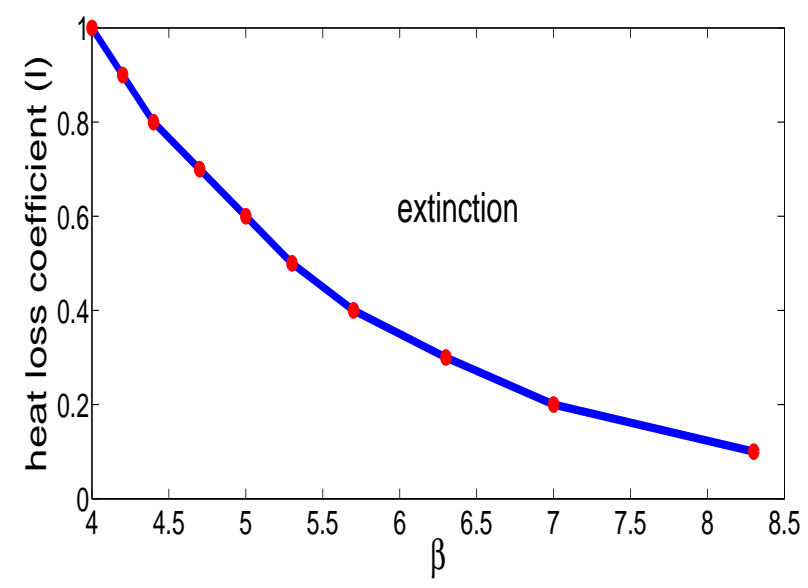

Figure 4. The locus of extinction points in the $(\beta, l)$ parameter space. Travelling waves do not exist above the curve.

We found that a convenient approach to determine the wave speed using our numerical schemes is to follow Towers et al. (2013), where the wave speed is calculated as

$$
c=\frac{\int_{0}^{L}|\partial v / \partial \tau| d \xi}{\int_{0}^{L}\|\nabla v\| d \xi},
$$


where $L$ is the length of the integration domain.

The wave speed for the adiabatic case depicted in figure 1 is approximately 6.887 obtained by using MOL and around 6.893 by FlexPDE ${ }^{\mathrm{TM}}$ respectively. On the other hand, the wave speed for the non-adiabatic case shown in figure 2 is about 6.855 (by MOL) and roughly 6.863 (by FlexPDE ${ }^{\mathrm{TM}}$ ). In all our numerical investigation we found that the wave speeds determined by MOL and FlexPDE ${ }^{\mathrm{TM}}$ are within \pm 0.01 of each other.

To study the effects of heat loss on the wave speed, we determine the combustion wave speeds as a function of $\beta$ for five distinctive values of $l$. This is shown in figure 3. Firstly, we note that the wave speed $c$ decays (grows) monotonically as we increase (decrease) $\beta$ in the feasible zone where there is a solution for the wave speed $c$. For those set of parameter values in the adiabatic case the solutions exists for any value of $\beta$ and its velocity decays gradually to zero as we increase $\beta$. Compared with the adiabatic case, there is a threshold value of $\beta$ which is similar with the one-step non-adiabatic reaction studied by Gubernov et al. (2004). When we choose a value greater than the critical value, the speed of the wave front drops to zero. For values of $\beta$ beyond the critical value, there does not exist any combustion waves. Additionally, the wave speed prior to the extinction value does not approach zero. For example, we found that no combustion wave was possible for $\beta>8.2$ for the case $l=0.1$ but the wave speed was around 1.15 with $\beta=8.2$. Hence, $\beta=8.2$ represents the extinction point for the case with $l=0.1$. Moreover, as expected, the wave speed for the case with heat loss is lower than that of the case under adiabatic conditions.

Finally, we investigate the location of the extinction point as a function of the heat loss coefficient $l$ for various values of $\beta$ for the non-adiabatic case. Figure 4 shows the locus of points representing the extinction values. The extinction value of $l$ decreases as we increase the value of the exothermic reaction. It is physically reasonable as increasing $\beta$ means the reaction is less exothermic. In other words, when the heat loss is large, then for the flame to propagate, the exothermic parameter should be sufficiently small (i.e. the fuel is more exothermic). For instance, no travelling wave is possible with $l=1$ when $\beta \geq 4$.

\section{CONCLUSIONS}

In this paper we undertook a preliminary investigation of a two-step competitive exothermic reactions model in a one-dimensional system with heat loss. The model was studied by solving the governing PDEs by using the method of lines which was independently validated using a commercial finite-element software, FlexPDE ${ }^{\mathrm{TM}}$. Solutions by MOL agree well with those using FlexPDE ${ }^{\mathrm{TM}}$. Then we compared solutions for the adiabatic case with those for the non-adiabatic case. We show that the wave speeds for the adiabatic case is larger than those for the case with heat loss. The location of extinction points was also determined for each value of the heat loss coefficient.

For simplicity, we only set the ambient temperature to be zero. As reported by Gubernov et al. (2005), we expect the variation of the ambient temperature to only have quantitative impact on the behaviour of the combustion wave. We also intend to investigate the behaviour of the solution for a wide range of values of Lewis number for different fuels. Gubernov et al. (2008) have shown that the variation of Lewis number has an impact on the behaviour of travelling waves. Towers et al. (2013) have shown the existence of a multiplicity of flame fronts propagating at different speeds for the same parameters under adiabatic limit. So in our future work we will look at how bi-stability will change when heat loss is included in the system. Sidhu et al. (2013) have shown the existence of pulsations for larger Lewis numbers. Another area for future work is to examine how pulsations will be affected with the inclusion of heat loss. Finally, the Evans function method will be employed to study the stability of the front, particularly to determine the cause of the onset of instabilities for the non-adiabatic case.

\section{REFERENCES}

Bechtold, J. and C. Law (1994). The structure of premixed methane-air flames with large activation energy. Combustion and flame 97(3), 317-338.

FlexPDE, P. Solutions inc. URL http://www. pdesolutions. com.

Gubernov, V., G. Mercer, H. Sidhu, and R. Weber (2004). Evans function stability of non-adiabatic combustion waves. In Proceedings of the Royal Society of London A: Mathematical, Physical and Engineering Sciences, Volume 460, pp. 2415-2435. The Royal Society. 
Z. Huang et al., Investigating Flame Fronts in Competitive Exothermic Reactions

Gubernov, V., H. Sidhu, and G. Mercer (2005). The effect of ambient temperature on the propagation of nonadiabatic combustion waves. Journal of mathematical chemistry 37(2), 149-162.

Gubernov, V., H. Sidhu, G. Mercer, A. Kolobov, and A. Polezhaev (2008). The effect of lewis number variation on combustion waves in a model with chain-branching reaction. Journal of Mathematical Chemistry 44(3), 816-830.

Martirosyan, N., S. Dolukhanyan, and A. Merzhanov (1983a). Experimental observation of the nonuniqueness of stationary combustion in systems with parallel reactions. Combustion, Explosion, and Shock Waves 19(6), 711-712.

Martirosyan, N., S. Dolukhanyan, and A. Merzhanov (1983b). Nonuniqueness of stationary states in combustion of mixtures of zirconium and soot powders in hydrogen. Combustion, Explosion, and Shock Waves 19(5), 569-571.

MATLAB ${ }^{\circledR}$. The MathWorks Inc.

Merzhanov, A. G. (1990). Self-propagating high-temperature synthesis: twenty years of search and findings. Combustion and plasma synthesis of high-temperature materials, 1-53.

Sánchez, A., G. Balakrishnan, A. Linán, and F. Williams (1996). Relationships between bifurcation and numerical analyses for ignition of hydrogenair diffusion flames. Combustion and flame 105(4), 569-590.

Schiesser, W. (1991). The Numerical Method of Lines: Integration of Partial Differential Equations. San Diego : Academic Press.

Seshadri, K., N. Peters, and F. Williams (1994). Asymptotic analyses of stoichiometric and lean hydrogen-air flames. Combustion and Flame 96(4), 407-427.

Sidhu, H., I. Towers, V. Gubernov, A. Kolobov, and A. Polezhaev (2013). Investigation of flame propagation in a model with competing exothermic reactions. In Chemeca 2013, Brisbane, 29 Sep 2013 - 02 Oct 2013., Brisbane, Australia, pp. 29350-1-29350-6.

Towers, I., V. Gubernov, A. Kolobov, A. Polezhaev, and H. Sidhu (2013). Bistability of flame propagation in a model with competing exothermic reactions. In Proceedings of the Royal Society of London A: Mathematical, Physical and Engineering Sciences, Volume 469, pp. 20130315. The Royal Society.

Towers, I., H. Sidhu, V. Gubernov, A. Kolobovb, and A. Polezhaevb (2013). Multiplicity in combustion wave behaviour for a model with competing exothermic reactions. In MODSIM2013, 20th International Congress on Modelling and Simulation, Adelaide, Australia, 01 Dec 2013 - 06 Dec 2013, pp. 99-105.

Weber, R., G. Mercer, H. Sidhu, and B. Gray (1997). Combustion waves for gases $(\mathrm{le}=1)$ and solids (le). In Proceedings of the Royal Society of London A: Mathematical, Physical and Engineering Sciences, Volume 453, pp. 1105-1118. The Royal Society.

Westbrook, C. K. and F. L. Dryer (1981). Simplified reaction mechanisms for the oxidation of hydrocarbon fuels in flames. Combustion science and technology 27(1-2), 31-43.

Williams, F. (1985). Combustion theory: the fundamental theory of chemically reacting flow systems. Menlo Park, California: Benjamin/Cummings.

Zeldovich, I., G. Barenblatt, V. Librovich, and G. Makhviladze (1985). The Mathematical theory of combustion and explosions. Consultants Bureau, New York. 